Note: This is a preprint of a paper being submitted for publication. Contents of this paper should not be quoted nor referred to without permission of the author(s).

\title{
Field Emission and Nanostructure of Carbon Films
}

V. I. Merkulov, ${ }^{\text {a) }}$ D. H. Lowndes, ${ }^{\text {a) }}$ and L. R. Baylor ${ }^{\text {b) }}$

Oak Ridge National Laboratory, Oak Ridge, TN 37831

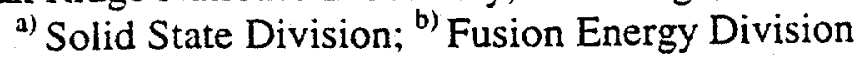

\begin{tabular}{c} 
Submitted to: \\
Amorphous and Nanostructured Carbon \\
Materials Research Society Fall Meeting, Boston, MA, 1999 \\
\hline
\end{tabular}

The submitted manuscript has been authored by a
contractor of the U.S. Government under contract DE-
ACUS-9hoR22464. Accortingly. the U.S. Government
retains a nonexclusive. coyalty-tree license to pubtish or
reproduce the published form of this contribution. or allow
uthers to do so, ior U.S. Government purposes."

November 1999

Prepared by

Solid State Division

Oak Ridge National Laboratory

P.O. Box 2008

Oak Ridge, Tennessee 37831-6056

managed by

LOCKHEED MARTIN ENERGY RESEARCH CORP.

for the

U.S. DEPARTMENT OF ENERGY

under contract DE-AC05-96OR22464 


\title{
FIELD EMISSION AND NANOSTRUCTURE OF CARBON FILMS
}

\author{
V. I. MERKULOV, ${ }^{\text {a) }}$ D. H. LOWNDES, , ${ }^{\text {a) }}$ AND L. R. BAYLOR ${ }^{\text {) }}$ \\ Oak Ridge National Laboratory, Oak Ridge, TN 37831, merkulov@solid.ssd.ornl.gov

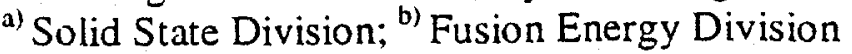

\begin{abstract}
The results of field emission measurements of various forms of carbon films are reported. It is shown that the films' nanostructure is a crucial factor determining the field emission properties. In particular, smooth, pulsed-laser deposited amorphous carbon films with both high and low sp3 contents are poor field emitters. This is similar to the results obtained for smooth nanocrystalline, $\mathrm{sp}^{2}$ - bonded carbon films. In contrast, carbon films prepared by hot-filament chemical vapor deposition (HF-CVD) exhibit very good field emission properties, including low emission turnon fields, high emission site density, and excellent durability. HF-CVD carbon films were found to be predominantly $\mathrm{sp}^{2}$-bonded. However, surface morphology studies show that these films are thoroughly nanostructured, which is believed to be responsible for their promising field emission properties.
\end{abstract}

\section{INTRODUCTION}

Electron field emission from various carbon-based materials recently has drawn significant attention of the industrial and scientific communities world wide, as potential applications of these materials in cold cathodes for field emission displays and other vacuum microelectronic devices appear feasible. Traditionally, cold cathodes have been made in the form of gated microtips because most materials, including $\mathrm{Mo}$ and $\mathrm{Si}$ which have been used in prototype devices, have a large $(-5 \mathrm{eV}$ ) work function (for metals) or electron affinity (for semiconductors). Consequently, a high electric field is required to extract electrons from these materials. The high electric field is achieved by fabricating microtips with a very sharp radius of curvature which leads to a high geometric enhancement factor (GEF) of the electric field. However, an alternative approach that eliminates complicated and expensive tip fabrication is to use flat cathodes made of a material with either very low electron affinity or intrinsic nanostructure which would provide sufficiently high GEF. For a practical emitter, used for example in a field emission display, many parameters must be taken into account, including low emission turn-on field, determined by the electron affinity or/and GEF, high emission site density, good stability, and long lifetime.

It has been established that diamond can possess a negative electron affinity surface ${ }^{2,3}$ which, in principle, should allow for electron emission under very low electric fields. Unfortunately, n-doping of bulk diamond is problematic and the transport of electrons to the conduction band, required for field emission applications, is difficult to achieve. As a result, despite its low electron affinity, single crystal diamond is not a good electron emitter. Recently, a few carbon (C) materials such as "coral-like" $\mathrm{C}^{4}, \mathrm{CVD}$ diamond", nanodiamond", and $\mathrm{C}$ nanotubes', were shown to exhibit very encouraging FE characteristics. The emission mechanism for these materials is not completely understood but seems to involve substantial GEF or perhaps electrical field enhancement created by highly non-uniform electronic properties over short (nanometer) distances. In this paper we report the results of FE tests of another $C$ material prepared by hot filament chemical vapor deposition (HF-CVD). This HF-CVD C seems promising for FE applications as it satisfies practically all of the cold cathode requirements 
outlined above. In particular, low turn-on voltages and high emission site density are observed which we attribute mainly to the nanostructure (and the high GEF associated with it) that is created during the deposition process, as opposed to during arcing or conditioning. Also, we discuss our FE results for tetrahedral amorphous carbon (ta-C) films as well as predominantly sp ${ }^{2}$ bonded $C$, since the results reported in the literature are mixed, with some researchers finding ta$C$ to exhibit very good FE properties ${ }^{8}$ while others ${ }^{4,9.10}$ do not.

\section{EXPERIMENT}

Amorphous $\mathrm{C}$ films with systematically variable tetrahedral $\left(\mathrm{sp}^{3}\right)$ bonding, ranging from amorphous diamond (or ta-C, up to $\sim 75 \% \mathrm{sp}^{3}$ ) to predominantly $\mathrm{sp}^{2}$-bonded amorphous $\mathrm{C}$ (a-C), were prepared by pulsed-laser deposition (PLD) in a high vacuum chamber with a base pressure of $3 \times 10^{-8}$ Torr using a Lambda Physik Compex 3011 pulsed excimer laser operated with ArF $(193 \mathrm{~nm})$. More details on the PLD experimental conditions can be found elsewhere ${ }^{11}$. HF-CVD $C$ was deposited in a vacuum chamber with a base pressure $<1 \times 10^{-7}$ Torr. A tungsten filament (0.3 mm diam.) was placed $\sim 5 \mathrm{~mm}$ above the sample. The current through the filament was 7 . Amps. The sample temperature during the deposition was $570-600^{\circ} \mathrm{C}$, as measured by a thermocouple attached directly to the sample surface. It is important to note that this temperature is below the softening point of glass substrates desirable for fabrication of cold cathodes for FEDs. Ethylene $\left(\mathrm{C}_{2} \mathrm{H}_{4}\right)$ was used as a gas source and was directed onto the filament using a stainless steel nozzle. The chamber pressure during the deposition was $1.2 \times 10^{-4}$ Torr. The deposition rate was $\sim 100 \AA / \mathrm{min}$, as determined by profilometry measurements. For both the PLD and HF-CVD films n-type (001)-oriented Si wafers were used as substrates.

Raman scattering measurements were made using a Lexel 3500 Ar ion laser operating at $514.5 \mathrm{~nm}$ and a Dilor XY Raman spectrometer equipped with a EG\&G OMA 4 CCD detector. Surface morphology studies were performed utilizing a Philips XL30/FEG high resolution scanning electron microscope (HRSEM) and a Nanoscope IIIa (Digital Instruments) atomic force microscope (AFM) that was operated in tapping mode.

Field emission measurements were carried out in a high vacuum chamber with a base pressure of $10^{-6}$ Torr. The measurements were taken by applying a positive voltage to a tungstencarbide current probe (anode) with tip diameter of $-25 \mu \mathrm{m}$ and by collecting electrons emitted from the samples tested (cathode). The current probe stage motion was computer controlled with the minimum step size of $75 \mathrm{~nm}$ in $\mathrm{x}-\mathrm{y}-\mathrm{z}$ directions, which allowed for precise control of the distance between the probe and the sample.

\section{RESULTS AND DISCUSSION}

First, it has to be emphasized that "conditioning" was required to obtain emission from all PLD amorphous C samples tested, regardless of their $\mathrm{sp}^{3}$ content. The conditioning typically resulted in arcing that occurred between the probe and the sample as the electric field was increased from 0 to $100-200 \mathrm{~V} / \mu \mathrm{m}$. After the conditioning, a typical turn-on field required to draw field emission current of $\sim \ln A$ from highly $\mathrm{sp}^{3}$-bonded $\mathrm{C}$ films (ta-C) was found to be $\sim$ $50 \mathrm{~V} / \mathrm{um}$ which is substantially higher than that obtained for other forms of carbon materials ${ }^{4-7}$. A typical emission current-applied electric field (I-E) curve for these films is shown in Fig.la. The macroscopic electric field, E, was simply calculated as the probe voltage divided by the probe-sample distance. Further, we find that conventional, predominantly $\mathrm{sp}^{2}-$ bonded a-C prepared by PLD with low $C$ ion kinetic energies exhibits very similar field emission characteristics, with even lower fluctuations of the emission current (see Fig. Ib). The emission curves follow the Fowler-Nordheim (FN) behavior as shown in the insets of Fig. $1 \mathrm{a}$ and $1 \mathrm{~b}$. 
Using a simplified FN equation ${ }^{12}$ and assuming the work function of graphite $(\Phi=4.6 \mathrm{eV})$ for amorphous $C$ films, it is possible to estimate the emission area (EA) and the geometric enhancement factor $(\beta)$ of the electric field due to sample's surface morphology. The calculations yield low $E A \sim 10^{-2} \mu \mathrm{m}^{2}$ and high $\beta \sim 100-150$ for both ta-C and $\mathrm{sp}^{2}$-bonded a-C films, contradictory to our AFM measurements which show that as deposited the films are extremely smooth ${ }^{13}$, with rms roughness $\sim 1-2 \AA$.
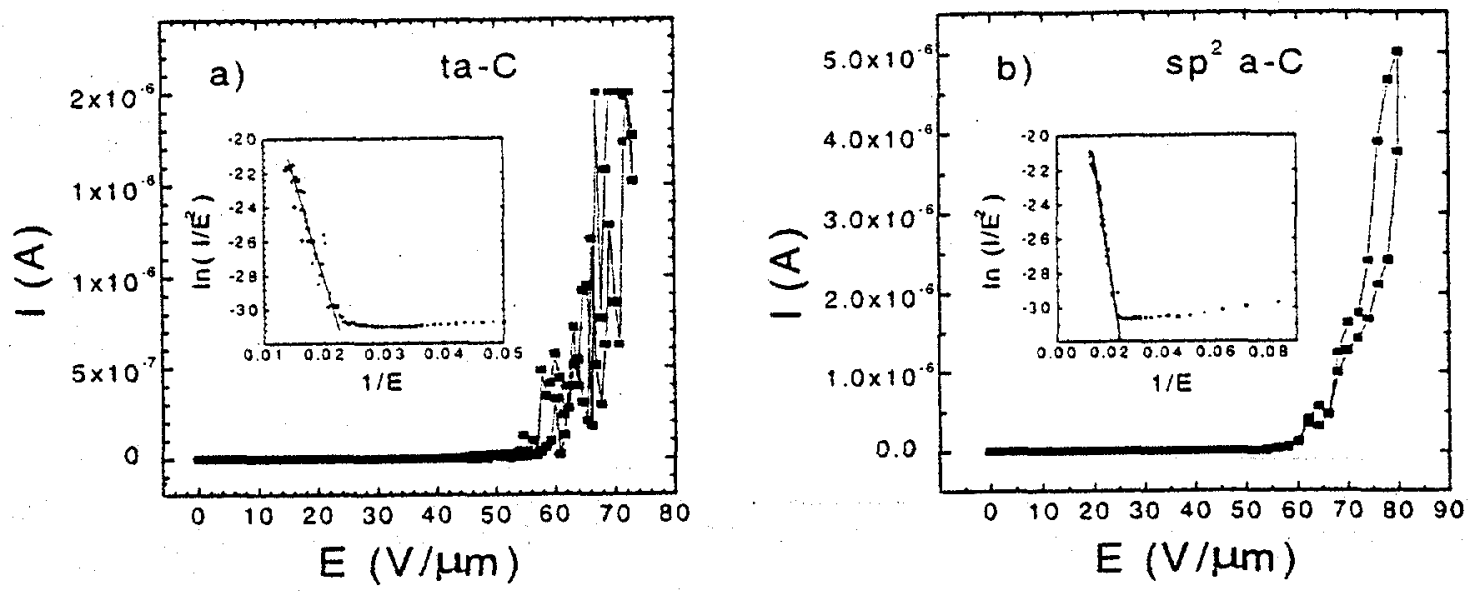

Figure 1. Emission current-applied electric field (I-E) curves and Fowler-Nordheim plots (insets) for $73 \% \mathrm{sp}^{3} \mathrm{ta}-\mathrm{C}(\mathrm{a})$ and predominantly $\mathrm{sp}^{2}$ bonded a-C (b) films.

SEM was employed to study changes in surface morphology that occurred after the arcing. The measurements reveal that for initially smooth films a crater of once-molten $\mathrm{C}$ film and $\mathrm{Si}$ substrate was formed at the location of the arcing (see Fig. 2). Given this fact and the FE characteristics of ta-C films described above, we conclude that $F E$ from ta-C is not due to its low electron affinity but rather should be attributed to the sharp protrusions around the crater formed during the arcing. The protrusions geometrically enhance the electric field around them, thereby providing for electron emission at moderate fields. These results are in good agreement with the observations of Talin et al. ${ }^{9}$, Coll et al. ${ }^{4}$ and Groning et al. ${ }^{14}$ but differ from those obtained by researchers who used parallel plate anode geometry in their experiments. We attribute this disagreement to the fact that the latter method has a drawback of "picking-up" the hottest spot on the sample and therefore provides no reliable information about the average FE properties of the

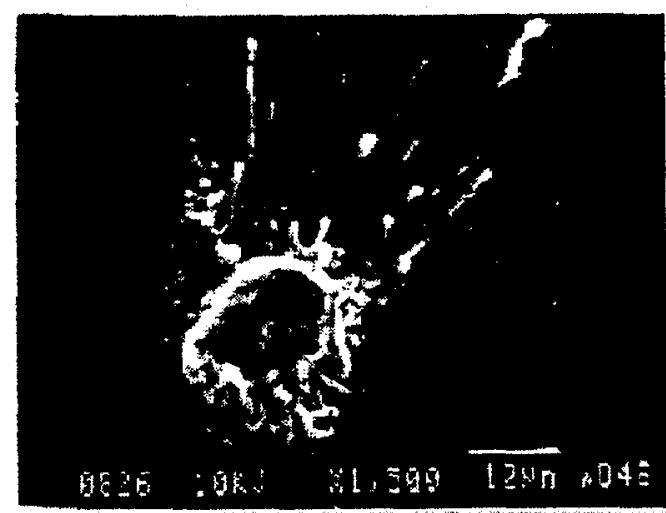

Figure 2. SEM image of a crater formed as a result of the conditioning process (arc discharge) of amorphous $\mathrm{C}$

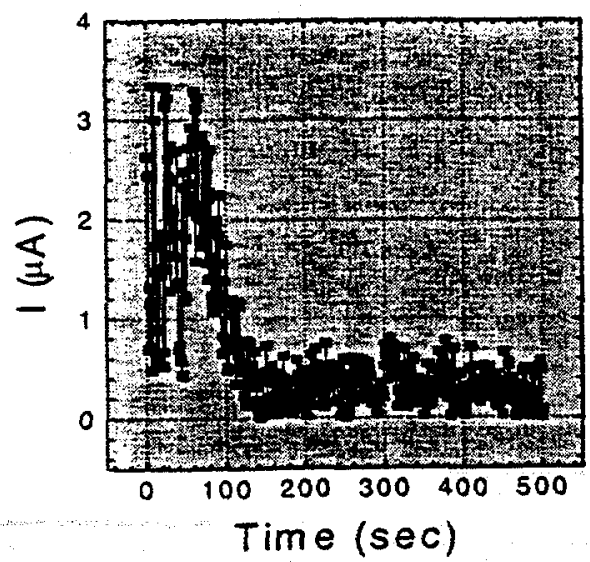

Figure 3. Instability of field emission from ta-C films. 
material over large surface areas. Also, in this method the leakage current across the spacers separating the anode from the cathode (sample) is rather difficult to distinguish from the true $\mathrm{FE}$ current. This is why we believe that only a point probe with three translational degrees of freedom or a phosphor screen (to directly image the emitting sites) should be used as the anode for FE measurements.

Finally, stability tests of FE from ta-C films were performed and a representative run is shown in Fig. 3. We have found the FE to be typically quite unstable and stop after a few minutes of operation. This can be attributed to the fact of the emission sites being quite sharp. As a result, they draw extremely high current densities and consequently burn out rather quickly. In addition, rather poor vacuum of $10^{-6}$ Tort may also be an issue as the ion bombardment during the FE measurements may severely damage and consequently terminate the emitting sites.

In contrast to PLD a-C films, HF-CVD C films exhibit very promising FE characteristics. First, no conditioning or arcing was required to initiate emission once the threshold voltage was reached and no substantial hysterisis was observed which suggests a non-destructive $\mathrm{FE}$ mechanism. Second, the turn-on voltage was quite low, varying from 9 to $30 \mathrm{~V} / \mu \mathrm{m}$. We also note that films deposited at higher $\mathrm{T}(-700 \mathrm{C})$ exhibited even lower turn-on fields $(\sim 7 \mathrm{~V} / \mu \mathrm{m})$. A typical I-E (emission current-applied electric field) curve is shown in Fig. 4a. The emission exhibits a little deviation from the Fowler-Nordheim behavior as shown in the inset. Third, we scanned the probe across the sample surface and found the emission site density (ESD) to be quite high. An example of a $2 \mathrm{D}(100 \mu \mathrm{m} \times 100 \mu \mathrm{m})$ scan is shown in Fig. $4 \mathrm{~b}$. The sample emitted everywhere across the surface, although the FE tum-on field varied from 9 to $23 \mathrm{~V} / \mu \mathrm{m}$. With the resolution limited by the size of the probe tip $(25 \mu \mathrm{m})$, we conclude that the ESD is at least 1 site/ $(25 \mu \mathrm{m})^{2}$ and probably much higher. The material is also found to be very robust. The maximum current obtained before damaging the sample was $-5-50 \mu \mathrm{A}$. This corresponds to a macroscopic current density of $\sim 1-10 \mathrm{~A} / \mathrm{cm}^{2}$ which is at least $10-100$ times higher than that required for FED applications. Finally, our preliminary results show that the emission is stable for at least 30-50 hours. Mechanical instabilities in our system precluded longer measurements of the emission lifetime.

It is interesting to elucidate the mechanisms responsible for such excellent FE properties of HF-CVD C. Raman scattering measurements were performed to get an idea about the
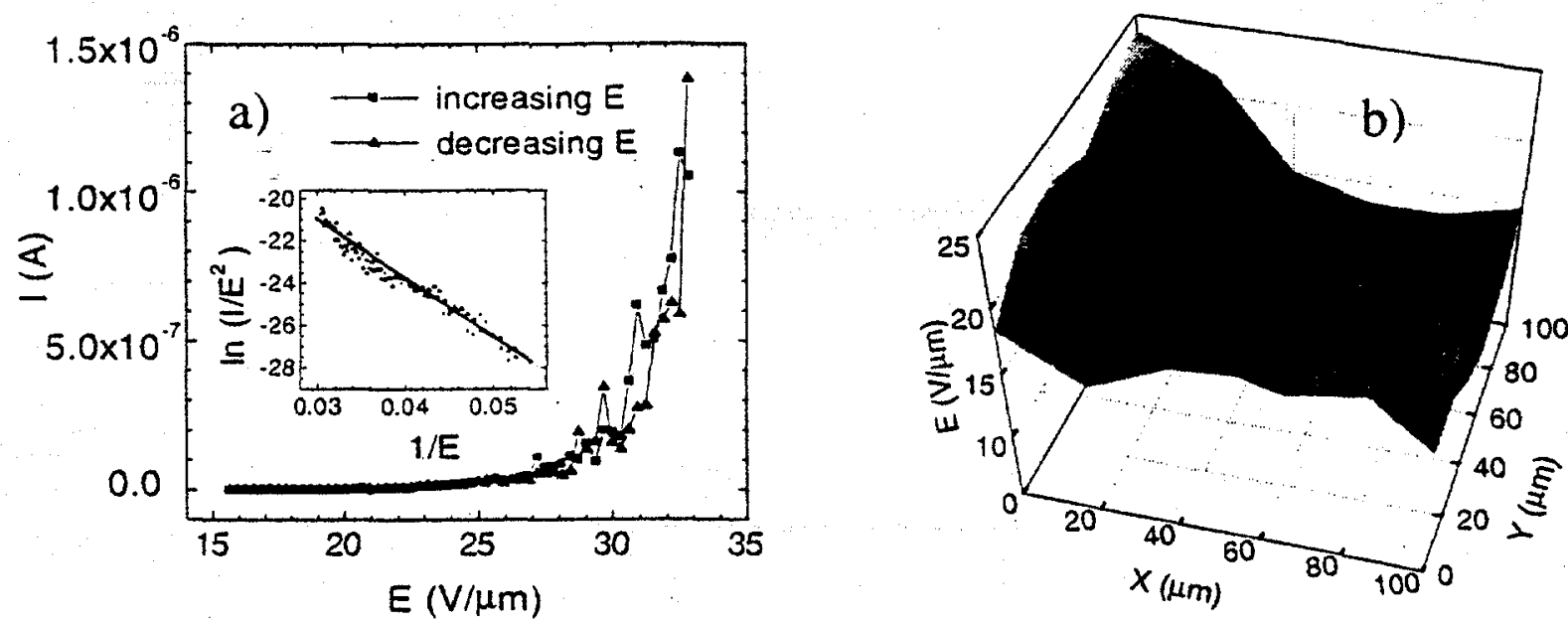

Figure 4. Emission current (I) - applied electric field (E) curve taken at a particular location (a) and emission tum-on field for $1 \mathrm{nA}$ current as a function of the probe position in the sample plane (b). The distance between the probe and the HF-CVD sample is $20 \mu \mathrm{m}$. 
structure of the films. The films were found to be quite opaque. The Si substrate peak at -520 $\mathrm{cm}^{-1}$ was not observed in the Raman response indicating metallic, $\mathrm{sp}^{2}$-bonded nature of HF-CVD $C$ films. The films also were found to be opaque in the infrared. A typical Raman spectrum of HF-CVD C is shown in Fig. 5 and consists of two broad peaks located at -1350 and $1600 \mathrm{~cm}^{-1}$. This is a characteristic spectrum of graphitic, nanocrystalline $C$ (nc-C) with a grain size $<25 \AA .15$ One can expect this kind of structure since our HF-CVD C was deposited at a relatively high substrate $\mathrm{T}\left(-600^{\circ} \mathrm{C}\right)$ which leads to the formation of nanocrystalline, $\mathrm{sp}^{2}$-bonded $\mathrm{C}$. However, we note that Raman scattering in the visible would not be able to detect small amounts of amorphous or nanocrystalline $\mathrm{sp}^{3}$-bonded $\mathrm{C}$ due to the low cross-section of the $\mathrm{sp}^{3}$-bonding in the visible. UV Raman scattering ${ }^{16,17}$, perhaps along with transmission electron microscopy (TEM) and electron energy loss spectroscopy (EELS), would be necessary to characterize HFCVD C more completely.

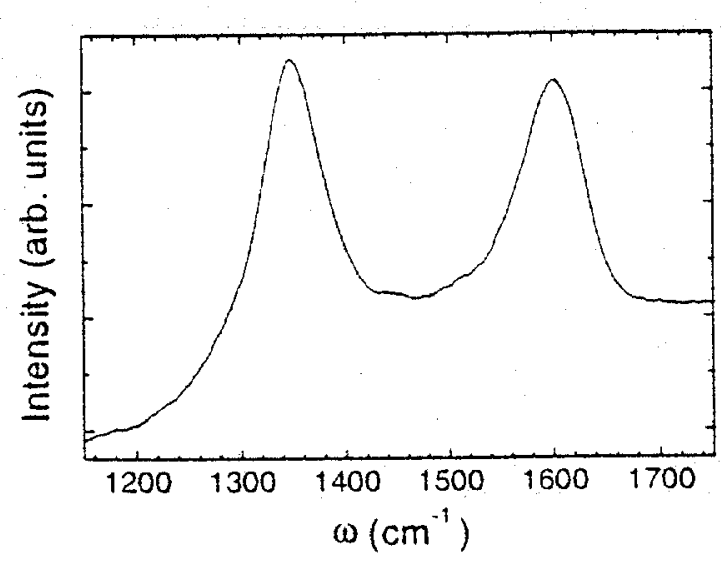

Figure 5. Raman spectrum of HF-CVD C. The two peaks located at $\sim 1350$ and $1600 \mathrm{~cm}^{-1}$ indicate $\mathrm{sp}^{2}$, nanocrystalline nature of the film.

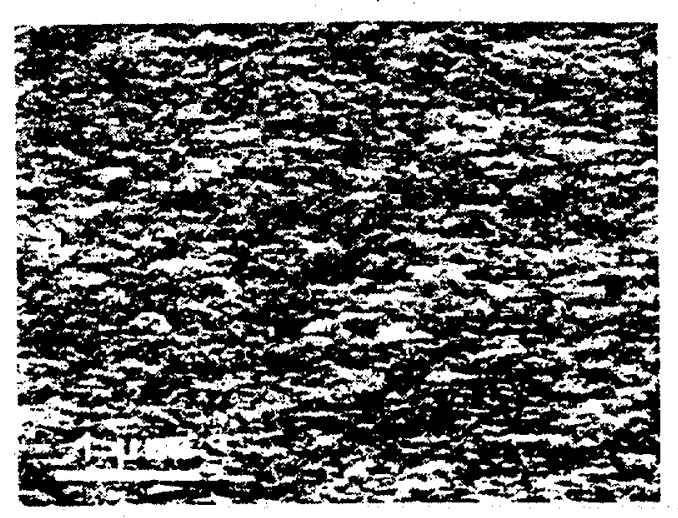

Figure 6. High resolution SEM image of HF-CVD C taken at $10 \mathrm{kV}$ and $50^{\circ}$ tilt angle.

High resolution SEM was utilized to investigate the surface morphology of the HF-CVD films. It was found that they are thoroughly nanostructured as depicted in Fig. 6. Many features in the $100 \mathrm{~nm}$ range and much smaller can be observed. We also note that smooth, nanocrystalline $C$ films deposited by PLD at the elevated substrate $T$ of $\sim 600-700 \mathrm{C}$ exhibited rather poor FE properties that were quite similar to those of PLD a-C. This is expected since graphite has a large work function and therefore a high FE turn-on field. From these comparisons we conclude that the excellent FE characteristics of HF-CVD C films are determined by their nanostructure and associated with it high GEF. In addition, a non-uniform H-termination of the $C$ surface and quantum size effects may also be among the possible contributing factors. The former may greatly increase the electric field through the tunneling barrier ${ }^{18}$ thereby promoting field emission at low voltages. The latter is manifested in the altered electronic structure as the size decreases. ${ }^{19}$ Consequently, the field emission properties of the material may change as well.

\section{CONCLUSIONS}

In conclusion, we have investigated the field emission properties of smooth and nanostructured C films prepared by PLD and HF-CVD correspondingly. While the former do not appear to be good field emission materials, the latter exhibit very promising FE characteristics, 
such as the absence of conditioning, low turn-on voltage, high emission site density and durability, which is attributed to their nanostructure.

\section{ACKNOWLEDGMENTS}

We are grateful to E. A. Kenik and the SHaRE facility for assistance with SEM measurements. We also thank $P$. H. Fleming for assistance with sample preparation. This research was partially sponsored by the Defense Advanced Research Projects Agency under contract DARPA-MIPR97-1357 with Oak Ridge National Laboratory (ORNL), and by the Office of Basic Energy Sciences, Division of Materials Sciences, U. S. Department of Energy. The research was carried out at ORNL, managed by Lockheed Martin Energy Research Corp. for the U. S. Department of Energy, under contract DE-AC05-960R22464.

\section{REFERENCES}

${ }^{1}$ C. A. Spindt, I Brodie, L. Humphrey, and E. R. Westerberg, J. Appl. Phys. 47, 5248 (1976).

${ }^{2}$ F. J. Himpsel, J. A. Knapp, J. A. Van Vechten, D. E. Eastman, Phys. Rev. B 20, 624 (1979).

${ }^{3}$ J. Van der Weide and R. J. Nemanich, Appl. Phys. Lett. 62, 1878 (1993).

${ }^{4}$ B. F. Coll, J. E. Jaskie, J. L. Markham, E. P. Menu, A. A. Talin, P. von All, in Covalently Bonded Disordered Thin-Film Materials, edited by M. P. Siegal, W. I. Milne, and J. E. Jaskie, (Mater. Res. Soc. Proc., Warrendale, 1998), p. 185.

${ }^{5}$ D. Zhou, A. R. Krauss, T. D. Corrigan, T. G. McCauley, R. P. H. Chang, and D. M. Gruen, J. Electrochem. Soc. 144, 224 (1997).

${ }^{6}$ W. Zhu, G. P. Kochanski, S. Jin, Science 282, 1471 (1998).

${ }^{7}$ W. A. de Heer, A. Chatelain, and D. Ugarte, Science 270, 1179 (1995).

${ }^{8}$ B. S. Satyanarayana, A. Hart, W. I. Milne, and J. Robertson, Appl. Phys. Lett. 71, 1430 (1997).

${ }^{9}$ O. Groning, O. M. Kuttel, E. Schaller, P. Groning, and L. Schlapbach, Appl. Phys. Lett. 69, 476 (1996).

${ }^{10}$ A. A. Talin, T. E. Felter, T. A. Friedmann, J. P. Sullivan, and M. P. Siegal, J. Vac. Sci. Tech. A 14, 1719 (1996).

"I V. I. Merkulov, D. H. Lowndes, G. E. Jellison, Jr., A. A. Puretzky, and D. B. Geohegan, Appl. Phys. Lett. 73, 2591 (1998).

${ }^{12}$ C. A. Spindt, I. Brodie, L. Humphrey, and E. R. Westerberg, J. Appl. Phys. 47, 5248 (1976).

${ }^{13}$ D. H. Lowndes, V. I. Merkulov, A. J. Pedraza, J. D. Fowlkes, A. A. Puretzky, D. B. Geohegan, and G. E. Gellison, Jr., in Surface Engineering: Science and Technology I, edited by A. Kumar, Y.-W. Chung, J. J. Moore, and J. E. Smugeresky, (The Minerals, Metals \& Materials Society, 1999), p. 113.

${ }^{14}$ O. Groning, O. M. Kuttel, E. Schaller, P. Groning, and L. Schlapbach, Appl. Phys. Lett. 69 , 476 (1996).

${ }^{15}$ R. J. Nemanich and S. A. Solin, Phys. Rev. B 20, 392 (1979).

${ }^{16}$ V. I. Merkulov, J. S. Lannin, C. H. Munro, S. A. Asher, V. S. Veerasamy, and W. I. Milne, Phys. Rev. Lett. 78, 4869 (1997).

${ }^{17}$ K. W. R. Gilkes, H. S. Sands, D. N. Batchelder, J. Robertson, and W. I. Milne, Appl. Phys. Lett. 70, 1980 (1997).

${ }^{18}$ J. Robertson, J. Vac. Sci. Tech. B 17, 659 (1999).

${ }^{19}$ G. P. Lopinski, V. I. Merkulov, and J. S. Lannin, Phys. Rev. Lett. 80, 4241 (1998). 